\title{
Stochastic job-shop scheduling: A hybrid approach combining pseudo particle swarm optimization and the Monte Carlo method
}

\author{
Kenta ARAKI* and Yasunari YOSHITOMI** \\ *Daikyo System Kaihatsu Co., Ltd. \\ 1-9-1 Edobori, Nishi-ku, Osaka 550-0002, Japan \\ ${ }^{* *}$ Graduate School of Life and Environmental Sciences, Kyoto Prefectural University \\ 1-5 Nakaragi-cho Shimogamo, Sakyo-ku, Kyoto 606-8522, Japan \\ E-mail: yoshitomi@kpu.ac.jp
}

Received 15 November 2015

\begin{abstract}
Many practical problems with uncertainties can be formulated as stochastic programming problems, and their optimal solutions are useful for decision-making. However, solving problems is generally difficult, and feasible methods for finding analytical solutions are needed. The purpose of this study is to propose a hybrid method that combines pseudo particle swarm optimization in an uncertain environment (PPSOUCE) and the Monte Carlo (MC) method for solving a stochastic programming problem. As an example, we used the proposed hybrid method to solve a stochastic job-shop scheduling problem (SJSSP). We compared our proposed PPSOUCE with the MC method to a hybrid method of a genetic algorithm in an uncertain environment (GAUCE) with the MC method. Numerical experiments illustrate that our method provides better solutions with shorter CPU times than those of the method that combines the GAUCE and the MC method.
\end{abstract}

Key words : Stochastic programming, Job-shop scheduling, Particle swarm optimization, Monte Carlo method

\section{Introduction}

Many practical problems with uncertainties can be formulated as stochastic programming problems, and their optimal solutions are useful for decision-making. However, solving these problems is generally difficult, and feasible methods for finding analytical solutions are needed.

To solve a stochastic programming problem, we assume that fluctuations in the environment are specified by the distribution functions of stochastic variables through all generations by a genetic algorithm (GA; Yoshitomi et al., 2000). We treat the stochastic fluctuation of the objective function and/or the constraint on the stochastic programming problem as a stochastic fluctuation of the fitness function in the GA. Since the fitness function expresses the fitness of an individual to that environment, the fitness function in the GA should fluctuate according to the distribution functions of the stochastic variables. We then analyze all solutions through all generations to obtain information about the optimum solution. We call this method the GA in an uncertain environment (GAUCE). By applying GAUCE to several stochastic programming problems, we confirmed that the individual that recurs most frequently through all generations gives the maximum expected value of the objective function (Yoshitomi et al., 2000).

First, we successfully applied the stochastic optimal assignment problem and the stochastic knapsack problem as preliminary examples for the verification and evaluation of GAUCE because these examples can be solved exactly by other methods (Yoshitomi et al., 2000). We then successfully applied GAUCE to the stochastic image-compression problem, a newly formulated problem that has recently received a great deal of attention in the field of computer and information science (Yoshitomi et al., 2000).

The job-shop scheduling problem (JSSP), which is an NP-hard problem, is one of the most difficult combinatorial optimization problems. However, since many scheduling problems in industry are formulated as JSSPs, a method for solving the JSSP has a practical as well as an academic importance. Thus, the JSSP has received considerable attention 
in the field of operations research.

In industrial applications, the stochastic job-shop scheduling problem (SJSSP) is more realistic than the JSSP. For example, in the real world, the processing time of a machine includes uncertainties. Therefore, these uncertainties need to be taken into consideration when scheduling or when formulating a scheduling problem such as the SJSSP. However, the SJSSP where many parameters, including, for example, the processing time of a machine, are treated as random variables is much more difficult to solve exactly than the JSSP where every parameter has a deterministic value. For this reason, much less research on the SJSSP than on the JSSP has been conducted.

In an earlier paper, we proposed a method for solving stochastic job-shop scheduling problems using hybrid of GAUCE and MC (Yoshitomi, 2002; Yoshitomi and Yamaguchi, 2003). GAUCE was applied to general SJSSP where the processing times are treated as stochastic variables. The several individuals having the highest frequencies through all generations were selected as the good schedules. The MC method was then used to determine the approximately optimal schedule among these good schedules.

Recently, in the operations research field, considerable attention has been paid to particle swarm optimization (PSO; Kennedy and Eberhart, 2001) as a method for solving optimal programming problems, including the JSSP (Lian, Jiao, and Gu, 2006; Xia and Wu, 2006; Moslehi and Mahnam, 2011) and the SJSSP (Zhang et al., 2012).

The purpose of this study is to propose a hybrid method that combines pseudo particle swarm optimization in an uncertain environment (PPSOUCE) and the MC method for solving the stochastic programming problem. The SJSSP is selected as an example for comparing the hybrid method of PPSOUCE with the MC method to the hybrid method of GAUCE with the MC method.

\section{Stochastic job-shop scheduling problem}

The SJSSP is a stochastic programming problem transformed from the JSSP. In actual industries, the processing time of a machine almost always has uncertainties. Therefore, these uncertainties must be taken into account when scheduling or formulating the scheduling problem as an SJSSP.

In this paper, the processing times of machines are treated as random variables, and the objective is to find a schedule that minimizes the expected value of the makespan. The other conditions of the SJSSP are the same as those of the regular JSSP. The SJSSP is formulated as follows:

1. We have $n$ jobs, $J_{1}, \ldots, J_{n}$, which are to be processed on $m$ machines, $M_{1}, \ldots, M_{m}$.

2. A machine can process only one job at a time.

3. The processing of a job on a machine is called an operation.

4. An operation cannot be interrupted.

5. A job consists of at most $m$ operations.

6. The sequence of operations within a job (the machine sequence) is given.

7. The processing times for operations are given as random variables that have stochastic distribution functions.

8. The operation sequences on the machines (the job sequences) are unknown.

9. On a Gantt chart, the positions of operations within a job are decided in order.

10. The starting time of an operation within a job should be as early as possible.

11. A schedule consists of the full set of job sequences.

12. The objective is to find a schedule that minimizes the expected value of the makespan.

In this study, a schedule is expressed by the job sequence on each machine. Item 10 in the above description implies that no operation can be postponed. The schedule that is uniquely represented by the above conditions is called a semi-active schedule, as described in the deterministic version (Yamada and Nakano, 1992); this means that the schedule is one in which no operation begins at an earlier time without altering the machining sequences.

An active schedule (Giffler and Thompson, 1969) is as follows (Yamada and Nakano, 1992). Consider a semi-active schedule and two operations $O_{j, i, r}$ and $O_{k, l, r}$ in that schedule that share the same machine $M_{r}$, where $j$ and $k$ denote the respective job numbers, $i$ and $l$ denote the positions of operation in the sequence of the job, 
and $r$ denotes the respective machine number. If $O_{k, l, r}$ is processed prior to $O_{j, i, r}$, and prior to processing $O_{k, l, r}$, machine $M_{r}$ has an idle period longer than $p_{j, i, r}$ (its processing time), then it is possible to reassign tasks so that operation $O_{j, i, r}$ is processed prior to $O_{k, l, r}$. Such reassigning is called a permissible left shift. A schedule having the property that no operation can be processed earlier by a permissible left shift is called an active schedule.

\section{PPSOUCE-MC approach}

\subsection{PSO and PPSOUCE}

In the PSO procedure, many particles move in multidimensional vector space. The position and the velocity of particle $i(i=1, \ldots, N)$ at moment $t$ are given by the vector $x_{i}(t)$ and the vector $v_{i}(t)$, respectively, where $N$ denotes the number of particles. The best position found thus far by the particle $i$ is expressed by a vector pbest ${ }_{i}(t)$; this gives the best value thus far for the evaluation function: $f\left(\right.$ pbest $\left._{i}(t)\right)$. The best position found thus far by the group having $n$ particles is expressed by the vector gbest $(t)$, which gives the best group value thus far for the evaluation function: $f($ gbest $(t))$.

To obtain the approximate optimal solution for the SJSSP, we modify the algorithm (Lian et al., 2006) introduced for the GA and used for the movement of a particle in the PSO. We also use the statistical method in GAUCE. We refer to the proposed method as the pseudo particle swarm optimization in an uncertain environment (PPSOUCE).

\subsection{Particle movement using the GA functions}

In the proposed method, the equation of motion of a particle is as follows:

$$
\begin{aligned}
& v_{i}^{k+1}=\text { pbest }_{j}^{k} \\
& \operatorname{child}_{i}^{k}(m, 0)=x_{i}^{k} \oplus v_{i}^{k+1}, m=1,2 \\
& \operatorname{child}_{i}^{k}(m, n)=\operatorname{Mn}\left(\operatorname{child}_{i}^{k}(m, 0)\right) \\
& \quad m=1,2, n=1,2, \ldots, N_{\max } \\
& x_{i}^{k+1}=\left\{\begin{array}{l}
x_{i}^{k}, \quad f\left(x_{i}^{k}\right) \leq f\left(\operatorname{child}_{i}^{k}(a, b)\right) \\
\operatorname{child}_{i}^{k}(a, b), f\left(x_{i}^{k}\right)>f\left(\operatorname{child}_{i}^{k}(a, b)\right)
\end{array}\right. \\
& f\left(\operatorname{child}_{i}^{k}(a, b)\right)=\text { min }_{1 \leq m \leq 2,0 \leq n \leq N_{\max }} f\left(\operatorname{child}_{i}^{k}(m, n)\right)
\end{aligned}
$$

where $i(i=1, \ldots, N)$ is the respective particle number, $j$ is the respective number of the particle selected randomly, $\oplus$ is the crossover operator, $\operatorname{child}_{i}^{k}(m, 0)$ is the child particle generated by the crossover, $M_{n}$ is the mutation operator, and $\operatorname{child}_{i}^{k}(m, n)(n \neq 0)$ is the child particle generated by the mutation, $m$ is the number of respective child particle, $n$ is the mutation index, and $k$ is the step index corresponding to a discrete time. In this study, the respective job number is used for expressing the element of a vector for a particle, the crossover is that proposed by Hirano (1995), and the mutation is the shift change proposed by Ono (1997).

\subsection{PPSOUCE-MC approach}

For each step, the processing time is given as the random number generated according to the stochastic distribution function for the processing time. The basic procedure of the proposed method is as follows.

Step 1: Initial information $\left(x_{i}^{0}, v_{i}^{0}\right)(i=1, \ldots, N)$ for each particle is randomly given.

Step 2: The following procedure of substeps [1] and [2] is repeated in numerical order by the preset loop frequency.

[1] A random number generated according to the stochastic distribution function is given for each random valuable. 
[2] The information $\left(x_{i}^{k}, v_{i}^{k}\right.$, pbest $\left._{i}\right)$ for each particle is updated according to Eqs. (1) through (5), stated in Section 3.2 .

Step 3: Using the MC method to calculate the approximate expected makespan, an approximately optimal solution is found; it is the solution with high frequency in the Step 2 loop that gives the minimum approximate expected makespan.

Here, $i(i=1, \ldots, N)$ is the respective particle number, and $k$ is the step index corresponding to a discrete time scale. The respective job number is used for expressing the element of the vector $x_{i}^{k}$ for a particle. The vector $v_{i}^{k}$ is given by the initial information $\left(x_{i}^{0}, v_{i}^{0}\right)$ and Eqs. (1) through (5) in Section 3.2.

We consider only active schedules at each step. When a semi-active schedule is generated in the PPSOUCE process, that schedule is changed to its active version by a permissible left shift. Because the number of active schedules for the problem may be huge, MC is applied for selecting the best solution among several good solutions with high frequency in the loop in Step 2.

\section{Numerical experiments and discussion}

\subsection{Development environment}

The development of this system and the experiments for evaluation of the proposed method were performed in the following environment: personal computer: DELL OPTIPLEX 780 (CPU: Intel Core 2 Duo E8400 3.00 GHz, RAM: 4.00 GB); OS: Microsoft Windows 7 Professional; Development language: Microsoft Visual C++ 2008 Express Edition.

\subsection{Conditions}

The data for the $6 \times 6,10 \times 10$, and $20 \times 5$ JSSPs, taken from Muth and Thompson (1963), are shown in Tables 1-3, respectively. The dataset includes the routing of each job on each machine and the processing time for each operation (in parentheses). Tables 1-3 give the data, which are well-known benchmarks.

Table $1 \quad 6 \times 6$ job-shop scheduling problem (Muth and Thompson, 1963)

\begin{tabular}{cllllll}
\hline Job & \multicolumn{5}{c}{ Operation routing (processing time) } \\
\hline 1 & $3(1)$ & $1(3)$ & $2(6)$ & $4(7)$ & $6(3)$ & $5(6)$ \\
2 & $2(8)$ & $3(5)$ & $5(10)$ & $6(10)$ & $1(10)$ & $4(4)$ \\
3 & $3(5)$ & $4(4)$ & $6(8)$ & $1(9)$ & $2(1)$ & $5(7)$ \\
4 & $2(5)$ & $1(5)$ & $3(5)$ & $4(3)$ & $5(8)$ & $6(9)$ \\
5 & $3(9)$ & $2(3)$ & $5(5)$ & $6(4)$ & $1(3)$ & $4(1)$ \\
6 & $2(3)$ & $4(3)$ & $6(9)$ & $1(10)$ & $5(4)$ & $3(1)$ \\
\hline
\end{tabular}

Table 2 10×10 job-shop scheduling problem (Muth and Thompson, 1963)

\begin{tabular}{ccccccccccc}
\hline Job & \multicolumn{7}{c}{ Operation routing (processing time) } \\
\hline 1 & $1(29)$ & $2(78)$ & $3(9)$ & $4(36)$ & $5(49)$ & $6(11)$ & $7(62)$ & $8(56)$ & $9(44)$ & $10(21)$ \\
2 & $1(43)$ & $3(90)$ & $5(75)$ & $10(11)$ & $4(69)$ & $2(28)$ & $7(46)$ & $6(46)$ & $8(72)$ & $9(30)$ \\
3 & $2(91)$ & $1(85)$ & $4(39)$ & $3(74)$ & $9(90)$ & $6(10)$ & $8(12)$ & $7(89)$ & $10(45)$ & $5(33)$ \\
4 & $2(81)$ & $3(95)$ & $1(71)$ & $5(99)$ & $7(9)$ & $9(52)$ & $8(85)$ & $4(98)$ & $10(22)$ & $6(43)$ \\
5 & $3(14)$ & $1(6)$ & $2(22)$ & $6(61)$ & $4(26)$ & $5(69)$ & $9(21)$ & $8(49)$ & $10(72)$ & $7(53)$ \\
6 & $3(84)$ & $2(2)$ & $6(52)$ & $4(95)$ & $9(48)$ & $10(72)$ & $1(47)$ & $7(65)$ & $5(6)$ & $8(25)$ \\
7 & $2(46)$ & $1(37)$ & $4(61)$ & $3(13)$ & $7(32)$ & $6(21)$ & $10(32)$ & $9(89)$ & $8(30)$ & $5(55)$ \\
8 & $3(31)$ & $1(86)$ & $2(46)$ & $6(74)$ & $5(32)$ & $7(88)$ & $9(19)$ & $10(48)$ & $8(36)$ & $4(79)$ \\
9 & $1(76)$ & $2(69)$ & $4(76)$ & $6(51)$ & $3(85)$ & $10(11)$ & $7(40)$ & $8(89)$ & $5(26)$ & $9(74)$ \\
10 & $2(85)$ & $1(13)$ & $3(61)$ & $7(7)$ & $9(64)$ & $10(76)$ & $6(47)$ & $4(52)$ & $5(90)$ & $8(45)$ \\
\hline
\end{tabular}


Table $320 \times 5$ job-shop scheduling problem (Muth and Thompson, 1963)

\begin{tabular}{cccccc}
\hline Job & \multicolumn{5}{c}{ Operation routing (processing time) } \\
\hline 1 & $1(29)$ & $2(9)$ & $3(49)$ & $4(62)$ & $5(44)$ \\
2 & $1(43)$ & $2(75)$ & $4(69)$ & $3(46)$ & $5(72)$ \\
3 & $2(91)$ & $1(39)$ & $3(90)$ & $5(12)$ & $4(45)$ \\
4 & $2(81)$ & $1(71)$ & $5(9)$ & $3(85)$ & $4(22)$ \\
5 & $3(14)$ & $2(22)$ & $1(26)$ & $4(21)$ & $5(72)$ \\
6 & $3(84)$ & $2(52)$ & $5(48)$ & $1(47)$ & $4(6)$ \\
7 & $2(46)$ & $1(61)$ & $3(32)$ & $4(32)$ & $5(30)$ \\
8 & $3(31)$ & $2(46)$ & $1(32)$ & $4(19)$ & $5(36)$ \\
9 & $1(76)$ & $4(76)$ & $3(85)$ & $2(40)$ & $5(26)$ \\
10 & $2(85)$ & $3(61)$ & $1(64)$ & $4(47)$ & $5(90)$ \\
11 & $2(78)$ & $4(36)$ & $1(11)$ & $5(56)$ & $3(21)$ \\
12 & $3(90)$ & $1(11)$ & $2(28)$ & $4(46)$ & $5(30)$ \\
13 & $1(85)$ & $3(74)$ & $2(10)$ & $4(89)$ & $5(33)$ \\
14 & $3(95)$ & $1(99)$ & $2(52)$ & $4(98)$ & $5(43)$ \\
15 & $1(6)$ & $2(61)$ & $5(69)$ & $3(49)$ & $4(53)$ \\
16 & $2(2)$ & $1(95)$ & $4(72)$ & $5(65)$ & $3(25)$ \\
17 & $1(37)$ & $3(13)$ & $2(21)$ & $4(89)$ & $5(55)$ \\
18 & $1(86)$ & $2(74)$ & $5(88)$ & $3(48)$ & $4(79)$ \\
19 & $2(69)$ & $3(51)$ & $1(11)$ & $4(89)$ & $5(74)$ \\
20 & $1(13)$ & $2(7)$ & $3(76)$ & $4(52)$ & $5(45)$ \\
\hline
\end{tabular}

To transform a JSSP to an SJSSP, the processing time is treated as a random variable. The stochastic distribution function of the processing time is a normal distribution having as the mean values the values in parentheses in Tables $1-3$. The standard deviation of the normal distribution is given as the ratio of the deviations to the mean. In general, in industrial applications, the deviation of the processing time from its mean value is not very large. Therefore, as conditions for the stochastic processing time, the ratios of the standard deviation to the mean value were selected as (i) 0 (deterministic), (ii) 0.1 , and (iii) 0.2 . The case where the ratio is 0 was selected for comparison between the JSSP and the SJSSP.

The normal distribution was used as the stochastic distribution function because it is one of the simplest distributions for computer simulations. When PPSOUCE-MC is applied in an industrial application, the most appropriate distribution should be used. However, since this study is the first step in applying PPSOUCE-MC to an SJSSP, it is appropriate to use a simple distribution for the stochastic distribution function.

In this experiment, as the conditions of PPSOUCE, the number of particles was set to 4000 , and the loop frequency was set to 700; these were chosen after trying various values in preliminary experiments. The averaged makespans for the solutions that had the 100 highest frequencies through all updates of information of the particles in PPSOUCE were obtained from 100,000 MC samples for each schedule. The number 100,000 was also determined by preliminary experiments. The MC method was used for selecting the best among several good solutions, all of which showed high frequency in all information updates in PPSOUCE.

In PPSOUCE, the problem transforms from a stochastic problem to a deterministic one at each update of information, as outlined in the following. First, a random number is generated for each processing time according to its stochastic distribution function. In this study, in the conventional method, the uniform random number is transformed to a normally distributed random number with the mean and standard deviation taken from Tables $1-3$ and the stochastic condition, respectively. The random number is then used to transform the stochastic problem into a deterministic problem each time the particle information is updated. For each processing time, the random number is generated independently. When the random number generated for a processing time is negative, the processing time is treated as 0 . In this case, the corresponding operation is not performed.

Negative or zero processing times come from using the normal distribution as the stochastic distribution function. The above treatment for negative or zero processing times may not be the best way to reflect the stochastic nature of the 
processing time when using PPSOUCE-MC in an industrial application. However, in this numerical example, because the ratios of the standard deviation to the mean value are from 0 to 0.2 , the probability of having a negative or zero processing time for each operation is from 0 to 0.0000003 . Therefore, this treatment of negative or zero processing times has little influence on the overall numerical results.

In the framework of PPSOUCE, a good solution is one with high frequency through all updates of the particle information. The expected value of the makespan for the solution is approximated by the averaged makespan given by the MC process, in which the makespan is calculated 100,000 times for each schedule.

We compare the results by our method for the SJSSP with those obtained by conventional methods (GAUCE-MC), and we also supply the results for the JSSP as a reference.

\subsection{Results and discussion}

Figs. 1-3 show the convergence of PPSOUCE for JSSPs and SJSSPs when using the best value of $N_{\max }$ for the approximately optimal solution on each condition of the stochastic processing time. Figs. 1-3 show the cases of $6 \times 6$, $10 \times 10$, and $20 \times 5$ for JSSP and SJSSPs, respectively. In all cases of $6 \times 6,10 \times 10$, and $20 \times 5$ SJSSPs and during all iterations, the fluctuations of both the average and the minimum makespans are increased by the increase of the stochastic fluctuation on the processing time.

Table 4 shows a comparison of the proposed method (PPSOUCE-MC) and the conventional methods (method I (GA) (Yamad and Nakano, 1992), method II (GAUCE-MC) (Yoshitomi and Yamaguchi, 2003), and method III (GAUCE-MC) (Furutani, 2009)). As shown in Table 4, the proposed method is superior to the conventional methods (II and III), in terms of both the average value of the makespan and the calculation time. On the approximately optimal solution, the superiority of the proposed method to the conventional method III is more remarkable in the case of $10 \times 10$ SJSSPs than that in the case of $6 \times 6$ SJSSPs. Therefore, the proposed method is more effective for finding out the approximately optimal solution in larger solution space, compared with the conventional method III. In addition, on the optimal solution, the superiority of the proposed method to the conventional method I is only shown in the case of $20 \times 5$ JSSP. Therefore, the proposed method is more effective for finding out the optimal solution in larger solution space, compared with the conventional method I. Accordingly, the higher ability for finding out the (approximately) optimal solution in larger solution space is the characteristic of the proposed method, compared with conventional methods (I and III). In other words, the proposed method has the higher ability of global search, compared with conventional methods (I and III). In addition, the proposed method is superior to the conventional method II, in terms of the average value of the makespan in the case of $6 \times 6$ SJSSPs. As a function of global search, Eqs. (1) and (3) in Section 3.2 have the important role in the proposed method.

Tables 5-7 show the approximately optimal solutions obtained by PPSOUCE-MC. In Tables 5-7, the solutions are expressed in the order of jobs processed by each machine. For example, 143625 in Table 5(a) means that $J_{1}, J_{4}, J_{3}, J_{6}, J_{2}, J_{5}$ are processed by $M_{1}$ in that order. In Tables 6 and 7, the name of each machine is representatively expressed in (a). In Table 6, job 10 is indicated by 0 . In Table 7, the respective job number is expressed by the vigesimal numeral system. In Tables 5-7, "Frequency" means the number of particles that correspond to the solution. In Tables 5-7, "rank" means the order of frequency. For example, "(1)" in Table 4(a) means that the corresponding particle has the highest frequency of any particle appearing in the loop of Step 2, described in the Section 3.3. In all cases of $6 \times 6,10 \times 10$, and $20 \times 5$ SJSSPs, the frequencies of the best solution are decreased by the increase of the stochastic fluctuation on the processing time. This tendency was also shown in the references using conventional methods (GAUCE-MC method II (Yoshitomi and Yamaguchi, 2003) for the case of $6 \times 6$ SJSSPs and GAUCE-MC method III (Furutani, 2009) for both cases of $6 \times 6$ and $10 \times 10$ SJSSPs). This tendency might suggest that it is more difficult to find out the approximately optimal solution having very low value of the approximate expected makespan in the case of bigger stochastic fluctuation on the processing time.

\section{Conclusions}

This paper proposed a method for solving the stochastic job-shop programming problem using a hybrid of PPSOUCE with the MC method. PPSOUCE was applied to general SJSSPs, where the processing times were treated as stochastic variables and the objective function was the expected value of the makespan. Solutions with very high 
frequency in all iterations were selected as good schedules. The MC method was then used to determine the approximate optimal schedule from these good schedules. We performed numerical experiments that proved that the proposed method is superior to the hybrid of GAUCE with the MC method in terms of both the approximately optimal solution and the required calculation time.
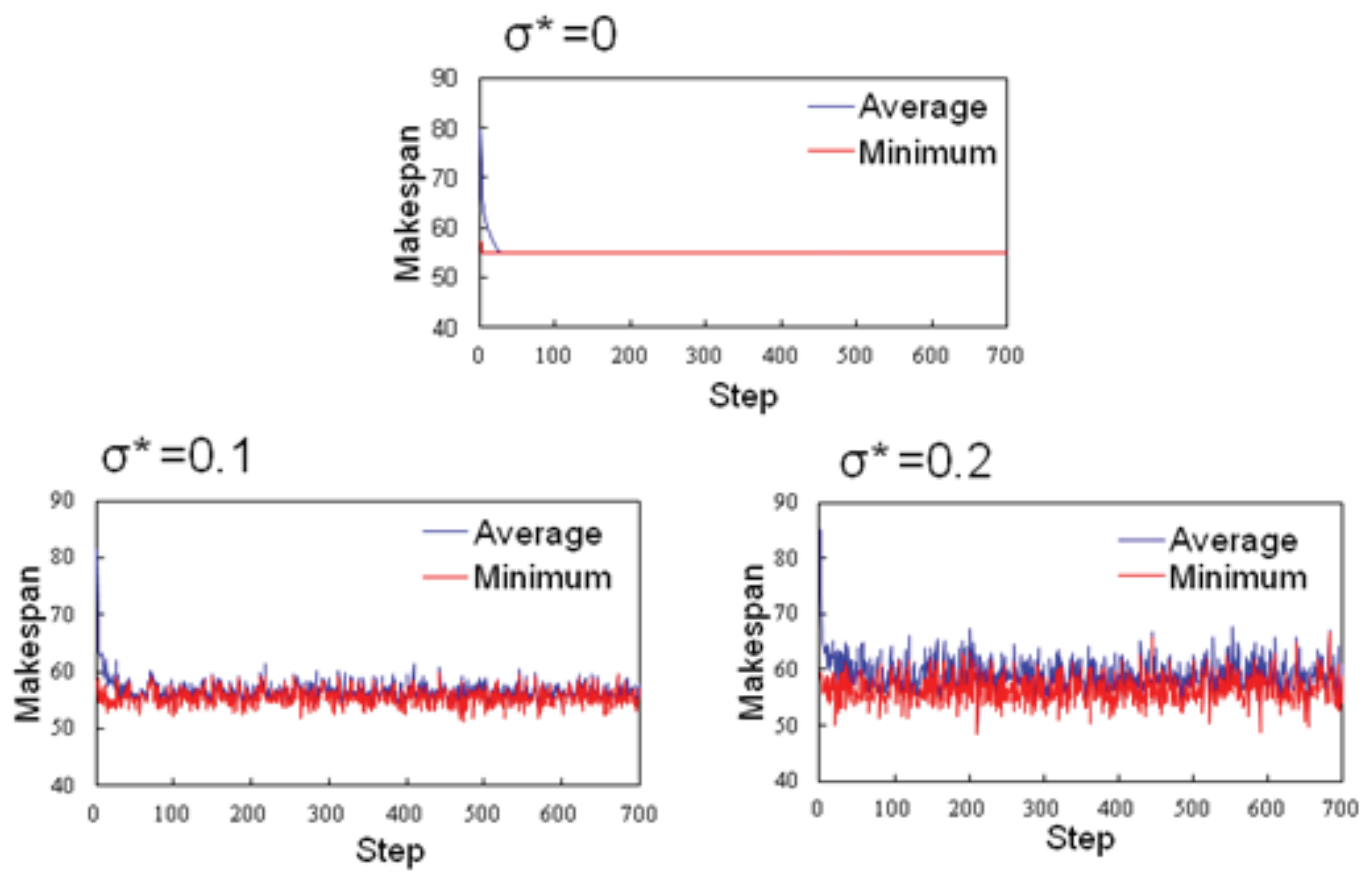

\section{$\sigma^{*}:$ Standard deviation /Average}

Fig. 1 Convergence of PPSOUCE for $6 \times 6$ JSSP and SJSSPs.
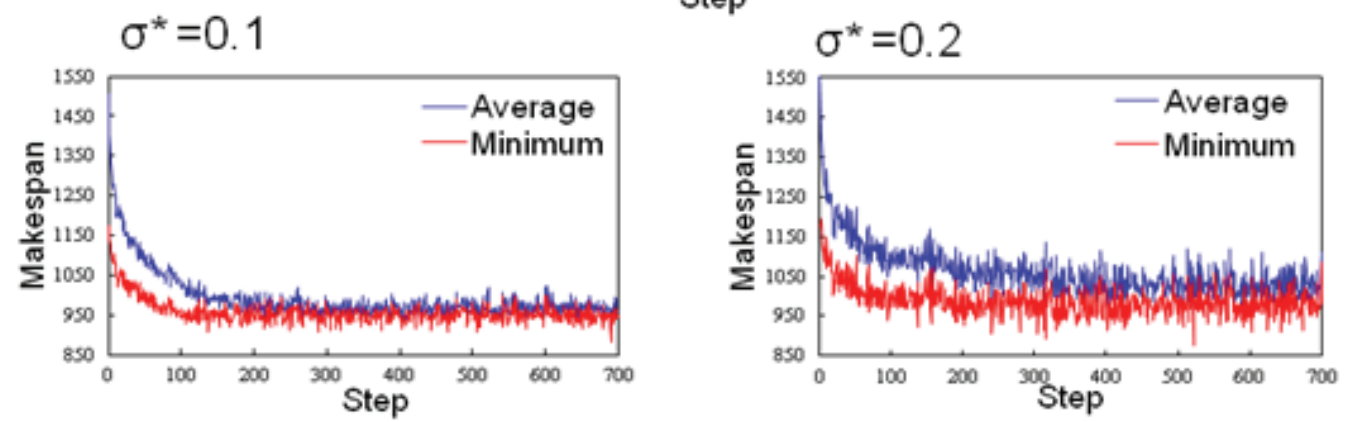

\section{$\sigma^{\star}:$ Standard deviation /Average}

Fig. 2 Convergence of PPSOUCE for $10 \times 10$ JSSP and SJSSPs. 

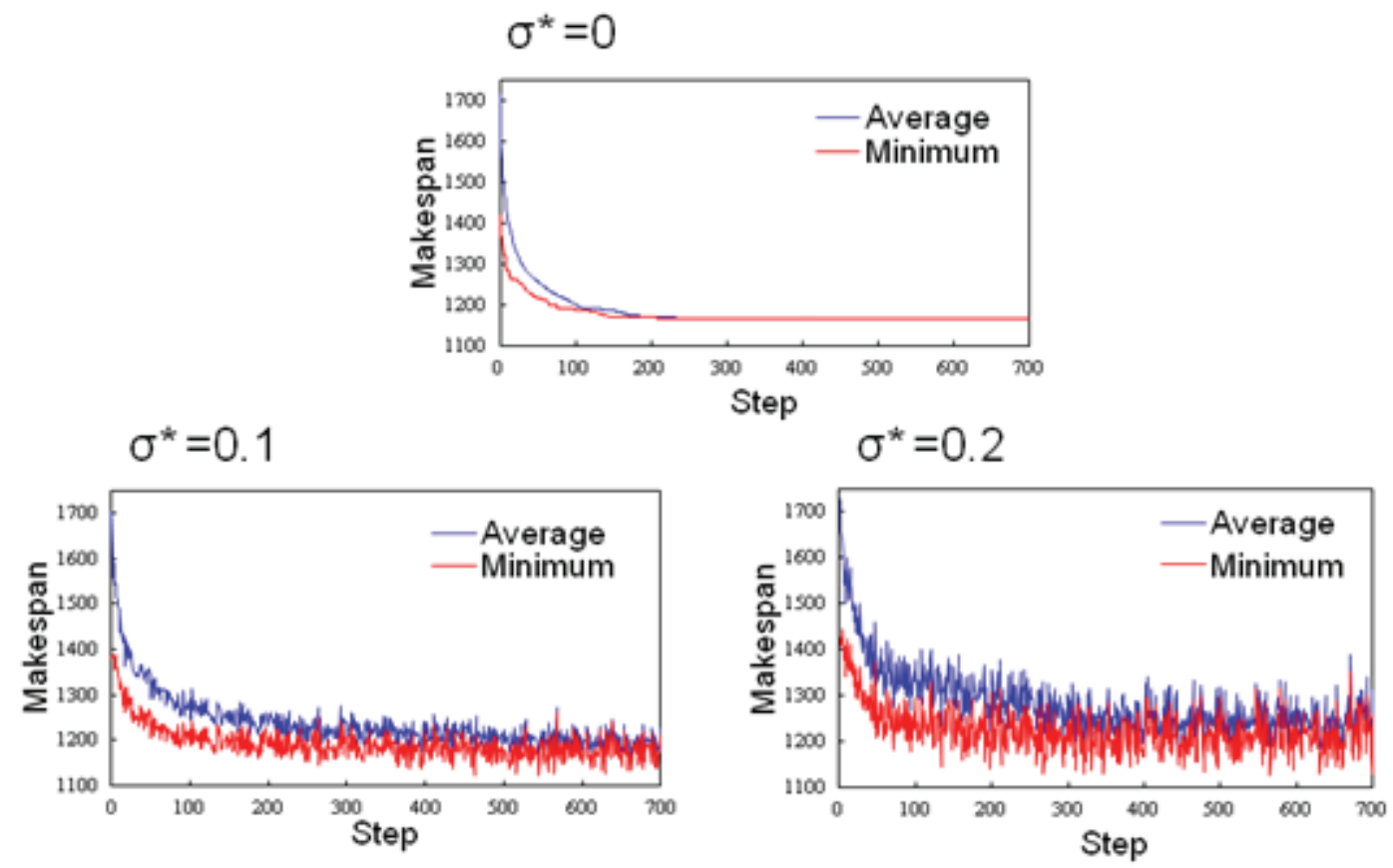

\section{$\sigma^{\star}:$ Standard deviation /Average}

Fig. 3 Convergence of PPSOUCE for $20 \times 5$ JSSP and SJSSPs.

Table 4 Comparison of PPSOUCE-MC and two versions of GAUCE-MC (a) Average value of makespan (rank)

\begin{tabular}{|c|c|c|c|c|}
\hline \multicolumn{2}{|c|}{ Standard deviation /Average } & 0 & 0.1 & 0.2 \\
\hline \multirow{5}{*}{$\begin{array}{c}6 \text { jobs, } \\
6 \text { machines }\end{array}$} & Minimum & 55 & - & - \\
\hline & GA & 55 & - & - \\
\hline & GAUCE-MC $^{\mathrm{a}}$ & $55(2)$ & $56.82(1)$ & $59.80(7)$ \\
\hline & GAUCE-MC $^{\mathrm{b}}$ & $55(1)$ & $56.11(1)$ & $58.04(20)$ \\
\hline & PPSOUCE-MC & $55(1)$ & 55.94(3) & $58.01(2)$ \\
\hline \multirow{4}{*}{$\begin{array}{c}10 \text { jobs, } \\
10 \text { machines }\end{array}$} & Minimum & 930 & - & - \\
\hline & GA & 930 & - & - \\
\hline & GAUCE-MC $^{b}$ & $977(1)$ & $996.95(11)$ & $1037.54(10)$ \\
\hline & PPSOUCE-MC & $930(1)$ & $958.39(27)$ & $1001.09(31)$ \\
\hline \multirow{3}{*}{$\begin{array}{c}20 \text { jobs, } \\
5 \text { machines }\end{array}$} & Minimum & 1165 & - & - \\
\hline & GA & 1184 & - & - \\
\hline & PPSOUCE-MC & $1165(1)$ & $1181.98(44)$ & $1228.59(27)$ \\
\hline
\end{tabular}

\begin{tabular}{|c|c|c|c|c|}
\hline \multicolumn{5}{|c|}{ (b) Calculation time (sec.) } \\
\hline \multicolumn{2}{|c|}{ Standard deviation /Average } & 0 & 0.1 & 0.2 \\
\hline \multirow{2}{*}{$\begin{array}{c}6 \text { jobs, } \\
6 \text { machines }\end{array}$} & GAUCE-MC $^{b}$ & 2080 & 3455 & 2884 \\
\hline & PPSOUCE-MC & 402 & 481 & 482 \\
\hline \multirow{2}{*}{$\begin{array}{c}10 \text { jobs, } \\
10 \text { machines }\end{array}$} & GAUCE-MC $^{\mathrm{b}}$ & 3851 & 18629 & 10742 \\
\hline & PPSOUCE-MC & 1270 & 1485 & 1491 \\
\hline $\begin{array}{c}10 \text { jobs, } \\
10 \text { machines }\end{array}$ & PPSOUCE-MC & 1647 & 1820 & 1848 \\
\hline
\end{tabular}

Notes:

GA: conventional method I (Yamada and Nakano, 1992)

GAUCE-MC ${ }^{\mathrm{a}}$ : conventional method II (Yoshitomi and Yamaguchi, 2003)

GAUCE-MC $^{\mathrm{b}}$ : conventional method III (Furutani, 2009) 
Table 5 Approximately optimal solutions obtained by PPSOUCE-MC (6×6 JSSP and SJSSP) (i) Standard deviation /Average $=0$ (deterministic), without mutation

\begin{tabular}{|c|c|c|c|c|c|c|c|}
\hline Frequency & \multicolumn{6}{|c|}{ Solution } & Makespan \\
\hline 1023191 & 143625 & 246153 & 312546 & 364125 & 254631 & 362514 & 55 \\
\hline \multicolumn{8}{|c|}{ (ii) Standard deviation /Average $=0.1, N_{\max }=4$} \\
\hline Frequency & \multicolumn{6}{|c|}{ Solution } & Average value of makespan \\
\hline 258626 & 143625 & 246531 & 312546 & 364125 & 253461 & 362514 & 55.94 \\
\hline \multicolumn{8}{|c|}{ (iii) Standard deviation / Average $=0.2, N_{\max }=5$} \\
\hline Frequency & \multicolumn{6}{|c|}{ Solution } & Average value of makespan \\
\hline 137795 & 143625 & 246513 & 312546 & 364125 & 254361 & 362514 & 58.01 \\
\hline
\end{tabular}

Table 6 Approximately optimal solutions obtained by PPSOUCE-MC (10×10 JSSP and SJSSP)

(i) Standard deviation /Average $=0$ (deterministic), $N_{\max }=6$

\begin{tabular}{|c|c|c|c|}
\hline Frequency & & & Makespan \\
\hline \multirow{5}{*}{928471} & $\mathrm{M}_{1}: 1297405863$ & $\mathrm{M}_{2}: 4670953812$ & \multirow{5}{*}{930} \\
\hline & $\mathrm{M}_{3}: 6458207913$ & $\mathrm{M}_{4}: 6795312408$ & \\
\hline & $\mathrm{M}_{5}: 4256819703$ & $M_{6}: 6597810324$ & \\
\hline & $\mathrm{M}_{7}: 4076981235$ & $M_{8}: 4659731820$ & \\
\hline & $M_{9}: 6405738912$ & $\mathrm{M}_{10}: 6279058431$ & \\
\hline \multicolumn{4}{|c|}{ (ii) Standard deviation /Average $=0.1, N_{\max }=4$} \\
\hline Frequency & \multicolumn{2}{|c|}{ Solution } & Average value of makespan \\
\hline \multirow{5}{*}{6034} & 9124570863 & 4679053812 & \multirow{5}{*}{958.39} \\
\hline & 6458207913 & 6975231408 & \\
\hline & 4258619703 & 6597821034 & \\
\hline & 4076928135 & 4597231860 & \\
\hline & 6405738912 & 6279058431 & \\
\hline \multicolumn{4}{|c|}{ (iii) Standard deviation /Average $=0.2, N_{\max }=8$} \\
\hline Frequency & \multicolumn{2}{|c|}{ Solution } & Average value of makespan \\
\hline \multirow{5}{*}{655} & 9214570863 & 4679053812 & \multirow{5}{*}{1001.09} \\
\hline & 6458207913 & 6975231408 & \\
\hline & 4256819703 & 6597821034 & \\
\hline & 4076928135 & 4597231860 & \\
\hline & 6405738912 & 6279054831 & \\
\hline
\end{tabular}

Table 7 Approximately optimal solutions obtained by PPSOUCE-MC $(20 \times 5$ JSSP and SJSSP) (i) Standard deviation /Average $=0$ (deterministic), $N_{\max }=10$

\begin{tabular}{cllc}
\hline Frequency & & Makespan \\
\hline \multirow{2}{*}{2996} & $\mathrm{M}_{1}:$ H5KGF2B1CJ9ID7A8E346 & $\mathrm{M}_{2}$ :G5HBKJF1A27CI6D843E9 & \\
& $\mathrm{M}_{3}:$ 5H8CKJ61AFDE2I973G4B & $\mathrm{M}_{4}: 5 H B G K J 912 C D A F 8 I 7 E 634$ & 1165 \\
& $\mathrm{M}_{5}:$ HHGBJKIC16D2A4938E7 & & \\
\hline
\end{tabular}

(ii) Standard deviation /Average $=0.1, N_{\max }=4$

\begin{tabular}{|c|c|c|c|}
\hline Frequency & \multicolumn{2}{|c|}{ Solution } & Average value of makespan \\
\hline \multirow{3}{*}{45} & 1F5HKIJD9GB2CA7E4368 & G51JKHBIFA6D7243CE89 & \\
\hline & 581HJK6CDAEI7F293B4G & 51HJBK9GDA2I7CE86F34 & 1181.98 \\
\hline & 51HIFKJBG6AD247C389E & & \\
\hline \multicolumn{4}{|c|}{ (iii) Standard deviation /Average $=0.2, N_{\max }=1$} \\
\hline Frequency & \multicolumn{2}{|c|}{ Solution } & Average value of makespan \\
\hline \multirow{3}{*}{5} & FH9JKG215CBDIAE63748 & JGHFB5K61A2CI3D7E489 & \\
\hline & 58HJ6CK1FAED29I7B34G & HJB95GK12AFCDE67I834 & 1228.59 \\
\hline & FHJ6B5GKIA1C2D473E89 & & \\
\hline
\end{tabular}




\section{References}

Furutani, T., A method for approximately solving large-scale stochastic job-shop scheduling problem using genetic algorithm in uncertain environment and Monte Carlo method, Master Thesis, Kyoto Prefectural University, Kyoto, Japan (2009) (in Japanese).

Giffler, B. and Thompson, G. L., Algorithm for solving production scheduling problem, Operations Research, Vol. 8 (1969), pp.164-176.

Hirano, H., Genetic algorithms with cluster averaging method for solving job-shop scheduling problems, Transactions of the Japanese Society for Artificial Intelligence, Vol. 10 (1995), pp.769-777.

Kennedy, J. and Eberhart, R. C., Swarm Intelligence, Morgan Kaufmann Publishers, San Francisco, California (2001).

Lian, Z., Jiao, B., and Gu, X., A similar particle swarm optimization algorithm for job-shop scheduling to minimize makespan, Applied Mathematics and Computation, Vol. 183 (2006), pp.1008-1017.

Moslehi, G. and Mahnam, M., A pareto approach to multi-objective flexible job-shop scheduling problem using particle swarm optimization and local search, International Journal of Production Economics, Vol. 129 (2011), pp.14-22.

Muth, J. F. and Thompson, G. L., Industrial Scheduling, Prentice-Hall, Englewood Cliffs, N.J. (1963).

Ono, I., Genetic algorithms for optimization taking account of characteristics preservation, Doctor Thesis, Tokyo Institute of Technology, Tokyo, Japan (1997) (in Japanese).

Xia, W. and Wu, Z., A hybrid particle swarm optimization approach for the job-shop scheduling problem, The International Journal of Advanced Manufacturing Technology, Vol. 29 (2006), pp.360-366.

Yamada, T. and Nakano, R., A genetic algorithm applicable to large scale job-shop problems, Proceedings of the Second International Conference on Parallel Problem Solving from Nature, (1992) pp.281-290.

Yoshitomi, Y., Genetic algorithm approach to solving stochastic job-shop scheduling problems, International Transactions in Operational Research, Vol. 9 (2002), pp.479-495.

Yoshitomi, Y., Ikenoue, H., Takeba, T., and Tomita, S., Genetic algorithm in uncertain environments for solving stochastic programming problem, Journal of the Operations Research Society of Japan, Vol. 43 (2000), pp. 266-290.

Yoshitomi, Y. and Yamaguchi, R., A genetic algorithm and the Monte Carlo method for stochastic job-shop scheduling, International Transactions in Operational Research, Vol. 10 (2003), pp.577-596.

Zhang, R., Song, S., and Wu, C., A two-stage hybrid particle swarm optimization algorithm for the stochastic job shop scheduling problem, Knowledge-Based System, Vol. 27 (2012), pp.393-406. 